\title{
Colectomía parcial laparoscópica electiva por enfermedad diverticular*
}

\author{
Drs. ERNESTO MELKONIAN T. ${ }^{1,2}$, MARCELO RODRÍGUEZ G. ${ }^{1}$, DAVID CONTRERAS F. ${ }^{1}$, \\ PATRICIO OPAZO S. ${ }^{1}$, IGNACIO ROBLES G. ${ }^{1}$, MAGDALENA BRAVO H. ${ }^{1}$
}

1 Servicio y Departamento de Cirugía Hospital del Salvador, Universidad de Chile.

2 Clínica Alemana.

Santiago, Chile.

\begin{abstract}
Elective laparoscopic colectomy for diverticular disease

Background: Colorectal colectomy is a less invasive thecnique that can be used for the treatment of diverticular disease, recently in younger patients. Aim: To report the experience with laparoscopic colectomy for diverticular disease. Material and Methods: All patients with indication for elective surgical resolution for diverticular disease were subjected to laparoscopic surgery from 1997 to 2009. The surgical protocols of these patients were analyzed. Results: Sixty patients with average age 53,8 (31 males) were operated in the period. Forty six were subjected to a sigmoidectomy and 14 to left hemicolectomy. Operative average time was 173 minutes and hospital stay 4,16 days. Three patients had to be converted to open surgery and three had complications that were managed without need of reoperation. During a median follow up of 38 months, the disease relapsed in two patients, which did not require a new intervention. Conclusions: Elective laparoscopic colectomy for diverticular disease is feasible and safe.
\end{abstract}

Key words: Laparoscopic surgery, colectomy, diverticular disease.

\section{Resumen}

La cirugía laparoscópica colorrectal, aunque de desarrollo lento, ha presentado en los últimos años ventajas con respecto a la cirugía abierta. Hay escasas publicaciones nacionales sobre esta técnica en enfermedad diverticular. Objetivo: Presentar nuestra experiencia en enfermedad diverticular en una serie consecutiva de pacientes con indicación quirúrgica electiva resueltos por vía laparoscópica. Material y Método: Se analizan los protocolos prospectivos de cirugía laparoscópica de colon con diagnóstico de enfermedad diverticular para resolución electiva, desde junio de 1997, hasta diciembre de 2009. Todo paciente con indicación quirúrgica electiva por esta patología fue resuelto por vía laparoscópica. Se estudian edad, sexo, tipo de resección, evolución postoperatoria inmediata y tardía. Resultados: Se operaron 60 pacientes, con edad promedio de 53,8 años. De éstos 46 fueron sigmoidectomías y 14 hemicolectomías izquierdas. El tiempo operatorio promedio fue de 173 minutos y la hospitalización de 4,16 días. Se convirtieron 3 pacientes $(5 \%)$ y hubo 3 complicaciones (5\%), que fueron manejadas médicamente. Hubo 1 estenosis de anastomosis tratada endoscópicamente como complicación tardía. Seguimiento promedio de 37,9 meses. Hubo 2 recidivas (3,3\%),

* Recibido el 25 de Mayo de 2010 y aceptado para publicación el 13 de Julio de 2010.

Correspondencia: Dr. Ernesto Melkonian T.

Salvador 364, Santiago, Chile. Fax: 5622699178

melkonianherve@entelchile.net 
tratadas médicamente y no hubo mortalidad en esta serie. Conclusión: La cirugía laparoscópica para la enfermedad diverticular electiva es factible de realizar en la gran mayoría de los pacientes, con baja morbilidad y rápida recuperación.

Palabras clave: Divertículos, colon, colectomía, laparoscopia.

\section{Introducción}

La cirugía laparoscópica de colon ha tenido un desarrollo lento desde su aparición en $1991^{1}$, con una larga curva de aprendizaje. Sin embargo, se han demostrado ventajas en sus resultados, siendo por esto cada vez más aceptada su aplicación, sobre todo en patología benigna ${ }^{2}$.

En nuestro medio hay, desde el año 2002, escasas series publicadas de colectomía laparoscópica para variadas patologías benignas ${ }^{3-5}$, excepto un centro, para enfermedad diverticular con resolución laparoscópica electiva ${ }^{6}$.

Por su parte, la enfermedad diverticular de colon, patología prevalente en pacientes de edad, ha presentado en los últimos años un aumento en su incidencia en pacientes más jóvenes, los que frecuentemente son de resolución quirúrgica ${ }^{7}$.

El objetivo de este trabajo es analizar los resultados de nuestra experiencia en una serie consecutiva de pacientes operados en forma electiva de enfermedad diverticular por vía laparoscópica.

\section{Material y Método}

Se analizan los protocolos llevados prospectivamente de cirugía laparoscópica de colon con diagnóstico de enfermedad diverticular para resolución electiva, desde junio de 1997 hasta diciembre de 2009.

Todo paciente que consultara electivamente por antecedente de una crisis de diverticulitis complicada o 2 o más crisis no complicadas fue resuelto por vía laparoscópica, esperándose al menos 45 días desde la última crisis. Se excluyeron pacientes que estuvieran cursando con una crisis de diverticulitis aguda clínica confirmada por TAC o ecografía abdominal.

En el estudio preoperatorio, a todos los pacientes se les realizó un enema baritado, al menos 45 días después de alguna crisis de diverticulitis, para evaluar la extensión de la enfermedad diverticular.

La preparación preoperatoria se realizó con 2 Fleet oral® el día previo a la operación y antibiótico profilaxis en la inducción anestésica con Ceftriaxona 1 gr. y Metronidazol $500 \mathrm{mg}$ iv.

La técnica consistió en confección de neumoperitoneo con aguja Veress a nivel umbilical, ubicación de dos trocares de 10-12 mm: uno a nivel umbilical y otro en fosa ilíaca derecha, dos de $5 \mathrm{~mm}$ : uno en flanco derecho y otro en flanco izquierdo. Excepcionalmente se utilizó un quinto trocar de $5 \mathrm{~mm}$, en caso de dificultad para liberar el ángulo esplénico.

Se utilizó abordaje lateral inicial en los primeros casos y posteriormente medial, realizando este último abordaje en la base del meso para visualizar con mayor facilidad el uréter izquierdo. Los vasos mesentéricos inferiores fueron ligados a nivel del tronco principal o en su primera bifurcación, sin necesidad de hacer una ligadura alta y se utilizó un stapler lineal vascular en la mayor parte de los casos, excepcionalmente clips y en los últimos casos Hemolok $^{\mathrm{R}}$.

Luego de liberar sigmoides y colon descendente, se marcó la zona proximal a seccionar con clip para no confundir este límite al extraer la pieza. El ángulo esplénico no fue liberado de rutina. Se seccionó el recto a nivel del promontorio y se extrajo la pieza operatoria por mini laparotomía suprapúbica. La anastomosis se realizó intracorpórea términoterminal con stapler circular 29, probando siempre la hermeticidad de la sutura con prueba hidroneumática. No se dejó drenaje de rutina.

Se definió como conversión la necesidad de realizar una laparotomía mayor de $5 \mathrm{~cm}$ para completar la operación.

Se analizaron edad, sexo, tipo de resección, evolución postoperatoria inmediata (antes de 30 días) y tardía.

El seguimiento fue clínico y por contacto telefónico.

\section{Resultados}

En ese período se operaron 193 pacientes de colon vía laparoscópica, de los cuales 60 fueron por enfermedad diverticular, siendo 31 hombres y 29 mujeres. La edad promedio fue de 53,8 años (37-77). Se realizaron 46 sigmoidectomías y 14 hemicolectomías izquierdas.

El tiempo operatorio fue de 173 minutos (120240) y la realimentación a líquidos un promedio de 1,2 días (Tabla 1). La hospitalización fue de 4,16 días (3-10), estando $75 \%$ de los pacientes de alta entre el tercer y cuarto día (Figura 1).

Se convirtieron a cirugía abierta 3 pacientes $(5 \%)$, uno por sangrado de mesorecto en el primer paciente de la serie, uno por fístula enterosigmoidea doble y uno por obesidad y mala visualización. 


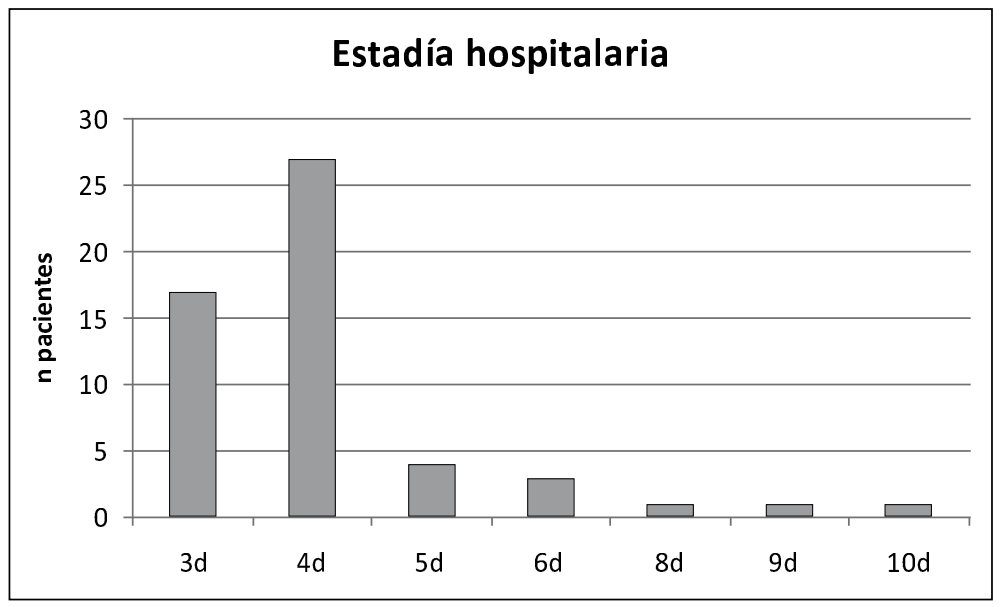

Figura 1. Tiempo de estadía hospitalaria postoperatorio.
Tabla 1. Tabla general

\begin{tabular}{|lll|}
\hline $\mathrm{n}$ & 60 pacientes & \\
Hombres & 31 \\
Mujeres & 29 & \\
Edad promedio & 53,8 & $(37-77)$ \\
Sigmoidectomía & 46 & \\
Hemicolectomía izq. & 14 & \\
Tiempo Op & 173 min & $(120-240)$ \\
Realimentación & 1,2 días & $(1-3)$ \\
Hospitalización & 4,16 días & $(3-10)$ \\
\hline
\end{tabular}

Tres pacientes (5\%) presentaron complicaciones precoces postoperatorias: un sangrado de anastomosis tratado con inyectoterapia endoscópica al cuarto día, un receso de anastomosis tratado con antibióticos a los 14 días y una neumonía.

En seguimiento promedio de 37,9 meses (2-144) hubo una estenosis de anastomosis como complicación tardía manifestada al año de la operación y tratada con éxito con dilatación endoscópica con balón. Hubo 2 recidivas $(3,3 \%)$ en pacientes con sigmoidectomía y divertículos en todo el colon, una al año y la otra a los 2 años de operado, siendo crisis no complicadas que se trataron médicamente.

No hubo mortalidad en la serie.

\section{Discusión}

La cirugía laparoscópica de colon, en los últimos años ha expandido su aplicación, sobre todo luego de demostrarse que en cáncer los resultados oncoló- gicos son similares a cirugía abierta ${ }^{8}$. Las ventajas de la técnica laparoscópica se han ido revelando tanto para patología benigna como maligna, una vez superada la curva de aprendizaje ${ }^{2,9}$. Se ha acortado la hospitalización a 3-4 días, como en nuestra y otras series, siendo de 5-7 días para cirugía abierta, asociado además a un menor íleo y dolor postoperatorios ${ }^{2,10}$.

A su vez, algunas series recientes han demostrado menor morbilidad precoz como neumonía e infección de herida ${ }^{2,11,12}$, lo que coincide con nuestra experiencia, con morbilidad precoz de 5\%, comparables con publicaciones, extranjera y nacional, que obtienen cifras de 4,5-15\% $\%^{6,13}$. Las 2 complicaciones no médicas de nuestra serie no requirieron de reoperación y fueron en relación a la anastomosis y no a la técnica laparoscópica, sin presentar ningún paciente infección de herida operatoria. El hecho de utilizar una mínima laparotomía y la menor alteración inmunitaria de este abordaje, probablemente influyen en esta baja tasa de infección de herida. El estudio de Duepree revela menos complicaciones tardías, hernia incisional y obstrucción intestinal, como consecuencia de una herida de menor tamaño $0^{14,15}$. La tasa de conversión de $5 \%$ en nuestra serie también es comparable con cifras internacionales que van de 6,6 a $19 \%^{10,16}$ para esta patología, debiendo considerarse que esta serie fue consecutiva, sin selección de pacientes. Creemos al respecto importante esperar el período de al menos 45 días luego de la última crisis para evitar la disección en planos inflamatorios y así disminuir la morbilidad y riesgo de conversión como lo confirma un estudio de Reissfelder ${ }^{17}$.

A su vez, la cifra de recidiva de 3,3\% está en los valores estudiados por Thaler de menos de $7 \%$, siendo importante para ésto no dejar divertículos re- 
siduales a distal, hecho que se asegura si se secciona el recto a nivel del promontorio sacro ${ }^{18}$.

La tasa de complicación tardía fue baja, manifestada por una estenosis anastomótica, complicación encontrada por Ambrossetti en un $17 \%$ de una serie de 68 pacientes de sigmoidectomía laparoscópica electiva por enfermedad diverticular ${ }^{19}$. En nuestras cirugías de colon no realizamos control endoscópico postoperatorio de rutina, método que pudiera detectar estenosis subclínicas. No hubo obstrucción intestinal ni hernias incisionales en la presente serie.

Pensamos que probablemente la colectomía parcial en estos pacientes, que han tenido crisis diverticulares repetidas o complicadas, logra ser realmente efectiva en bajar el riesgo de nuevos episodios; en la serie aparecen 2 crisis en 60 pacientes en un período de seguimiento promedio de más de 3 años.

Aunque esta serie no es comparativa, los resultados de recuperación rápida, baja morbilidad y evidente mejor resultado estético hacen difícil en la actualidad hacer estudios comparativos de cirugía laparoscópica en relación a la abierta, no habiendo series aleatorizadas en la literatura para enfermedad diverticular ${ }^{20}$.

En nuestra experiencia, sólo se ha realizado el abordaje laparoscópico para resolver la patología diverticular electiva desde 1997. La patología diverticular de colon por su parte ha presentado un aumento en gente joven que frecuentemente se resuelve quirúrgicamente, lo que es avalado por el promedio de edad de 53 años de nuestra experiencia ${ }^{7}$. Si bien según las últimas guías de práctica clínica de la Sociedad Americana de Cirugía Colorrectal, el número de crisis no complicadas no necesariamente marca en la actualidad la necesidad de operación, en dichas guías se menciona una mayor tendencia a operar pacientes jóvenes, por proyectarse en ellos un plazo más amplio para presentar nuevas crisis ${ }^{21}$.

Como conclusión podemos plantear que la cirugía laparoscópica electiva para la enfermedad diverticular es factible de realizar en la gran mayoría de pacientes como se muestra en esta serie consecutiva. Las bajas cifras de morbimortalidad precoz y tardía permiten ofrecer esta técnica como un método seguro para la resolución de la enfermedad diverticular de colon con indicación de cirugía electiva.

\section{Referencias}

1. Jacobs M, Verdeja JC, Goldstein HS. Minimally invasive colon resection (laparoscopic resection). Surg Laparosc Endosc. 1991;1:144-50.

2. Schwenk W, Haase O, Neudecker J, Muller J. Short term benefits for laparoscopic colorectal resection. Cochrane Database Syst Rev. 2005;20,3: CD003145.
3. Melkonian E, Wainstein C, Díaz H, Villar V, Campaña G, Parada H. Cirugía laparoscópica colorectal. Rev Chil Cir. 2004;56:107-11.

4. López F, Soto G, Zárate A, Pinedo G, Pérez G, Avendaño $R$, et al. Protocolo de cirugía laparoscópica intestinal. Rev Chil Cir. 2003;55:225-31.

5. Marín A, Vergara J, Espíndola L, Sepúlveda R, Urbano N. Cirugía colorrectal por videolaparoscopia en el Hospital Militar de Santiago. Rev Chil Cir. 2002; 54:595-9.

6. López-K F, Zárate A, Pinedo G, Molina M, Kronberg U, Pardo J. Resultados de la cirugía laparoscópica en el tratamiento electivo de la enfermedad diverticular de colon. Rev Med Chile 2008;136:594-9.

7. West SD, Robinson EK, Delu AN, Ligon RE, Kao LS, Mercer DW. Diverticulitis in the younger patients. Am J Surg. 2003;186:743-6.

8. Clinical Outcomes of Surgical Therapy Study Group. A Comparison of laparoscopically assisted and open colectomy for colon cancer. N Eng J Med. 2004;350:20509.

9. Guller U, Jain N, Hervey S, Purves H, Pietrobon R. Laparoscopic vs open colectomy: outcomes comparison based on large nationwide databases. Arch Surg. 2003;138:1179-86.

10. Senagore AJ, Duepree HJ, Delaney CP, Dissanaike S, Brady KM, Fazio VW. Cost structure of laparoscopic and open sigmoid colectomy for diverticular disease: similarities and differences. Dis Colon Rectum 2002;45:485-9.

11. Senagore AJ ,Madbouly KM, Fazio VW, Duepree HJ, Brady KM, Delaney CP. Advantages of laparoscopic colectomy in older patients. Arch Surg. 2003;138:252-6.

12. Alves A, Panis Y, Slim K, Heyd B, Kwiatowski F, et al. French multicentre prospective observational study of laparoscopic versus open colectomy for sigmoid diverticular disease. Br J Surg. 2005;92:1520-5.

13. Garrett KA, Champagne BJ, Valerian BT, Peterson D, Lee EC. A single training center's experience with 200 consecutive cases of diverticulitis: can all patients be approached laparoscopically? Surg Endosc. 2008;22:2503-8.

14. Duepree HJ, Senagore AJ, Delaney CP, Fazio VW. Does means of access affect the incidence of small bowel obstruction and ventral hernia after bowel resection? Laparoscopy versus laparotomy. J Am Coll Surg. 2003;197:177-81.

15. Wichmann M, Huttl T, Winter H, Spelsberg F, Angele $\mathrm{M}$, Heiss $\mathrm{M}$, et al. Immunological effects of laparoscopic vs open colorectal surgery. Arch Surg. 2005;140:692-7.

16. Dviwedi A, Chahin F, Agrawal S, Chau WY, Tootla A, Tootla F, et al. Laparoscopic colectomy vs open colectomy for sigmoid diverticular disease. Dis Colon Rectum 2002;45:1309-15.

17. Reissfelder C, Buhr HJ, Ritz JP. What is the optimal time of surgical intervention after an acute attack of 
sigmoid diverticulitis: early or late elective laparoscopic resection? Dis Colon Rectum 2006;49:1842-8.

18. Thaler K, Weiss EG, Nogueras JJ, Arnaud JP, Wexner Sd, Bergamaschi R. Recurrence rates at minimum 5year follow-up: laparoscopic versus open sigmoid resection for uncomplicated diverticulitis. Surg Laparosc Endosc Percutan Tech. 2003;13:325-7.

19. Ambrosetti P, Francis K, De Peyer R, Frossard JL. Colorectal anastomotic stenosis after elective laparoscopic sigmoidectomy for diverticular disease: a pros- pective evaluation of 68 patients. Dis Colon Rectum 2008;51:1345-9.

20. Purkayastha S,Constantinides VA, Tekkis PP, Athanasiou T, Aziz O, Tilney H, et al. Laparoscopic vs. open surgery for diverticular disease: a meta-analysis of nonrandomized studies. Dis Colon Rectum 2006;49:446-63.

21. Rafferty I, Shellito P, Hyman N, Buie D and the Standards Committee of the American Society of Colon and Rectal Surgeons. Practice Parameters for sigmoid diverticulitis. Dis Colon Rectum 2006;49:939-44. 